\title{
The Microwave Spectrum, Dipole Moment and Low Frequency Vibrational States for Phosphabenzene
}

\author{
Robert L. Kuczkowski and Arthur J. Ashe, III \\ Department of Chemistry, University of Michigan, Ann Arbor, Michigan 48104
}

\begin{abstract}
The microwave spectrum of the ground vibrational state and seven lowfrequency vibrational states for phosphabenzene $\left(\mathrm{C}_{5} \mathrm{H}_{5} \mathrm{P}\right)$ have been assigned. The small positive inertial defect and the alternation of intensities due to nuclear spin statistics for the ground state confirm a plannar structure with $C_{2 v}$ symmetry. A PC bond distance of $1.70-1.73 \check{A}$ and (CPC of $101^{\circ}-104^{\circ}$ were estimated based on plausible assumptions for the other ring parameters. Four low-frequeney fundamental modes of vibration were identified and vibrational assignments discussed. The nuclear spin statistics indicate that three of these modes were antisymmetric to the $\mathrm{C}_{2}$ axis while one mode was symmetric. The dipole moment has been determined as $1.54 \pm 0.02 \mathrm{D}$.
\end{abstract}

\section{INTRODUCTION}

Although benzene and pyridine are familar to all chemists the phosphorus analog called phosphabenzene (phosphorin) has only recently been synthesized (1). A small amount of the material was available for the investigation of its microwave spectrum. The original purpose of the study was to determine the structure and dipole moment of phosphabenzene in order to elucidate the extent of aromaticity. While the dipole moment was evaluated, the structural investigation was less successful since data on the less abundant isotopic species could not be obtained. However, the symmetry and planarity of the compound have been sstablished and some information on the P' $\mathrm{C}$ bound length and CPC angle was obtained. In addition a rich vibrational satellite spectrum was observed and assigned. This has provided information about the symmetries, frequencics and atom motions for four of the lowest modes of vibration.

\section{EXPERIMENTAL}

The transitions were measured at room temperature with a Stark-modulated spectrometer (2). The assignment of the ground state and the two strongest vibrational satellites were made by observing the Stark cffects using oscilloscope display. The weaker vibrational satellites' transitions were assigned from recorder traces obtained at several voltages. The uncertainties in the frequency measurements were estimated as $\pm 0.25 \mathrm{MHz}$. This range includes the weak 
vibrational satellite transitions for which it was difficult to reliably locate the center frequency.

The compound was prepared by the reaction of 1,4-dihydro-1,1-dibutyl stannabenzenc with phosphorus tribromide (1). The synthesis was not efficient for the preparation of enriched isotopic species, which made a complete structure determination contingent upon assigning ${ }^{13} \mathrm{C}$ species in natural abundance. Based on the intensities of the normal isotopic species, these transitions were estimated to be beyond the sensitivity of the spectrometer. An unsuccessful search was mad' for them using recorder techniques and a computer of average transients. The search for weaker lines was handicapped by the tendency of the compound to be quickly absorbed on the cell walls.

Intensity measurements were made several ways (3). Some information was obtained from the recorder traces. These data were useful for establishing the nuclear spin statistics, particularly in those regions where transitions of different symmetry fell close together. The traces were somewhat less useful for estimat-

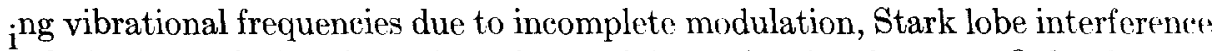
nd the intrinsic low intensity of the higher vibrational states. Only the two aowest vibrations were intense enough for quantitative relative intensity measurements. Besides estimating the relative intensities from the traces of several frequency regions, two transitions $\left(4_{22} \rightarrow 5_{23}\right.$ and $\left.4_{31} \rightarrow 5_{32}\right)$ were studied by using oscillosope display on the Michigan spectrometer and by the nonsaturation technique on the Hewlett-Packard spectrometer at Michigan State University. The uncertainty estimates for the vibrational frequencies were nevertheless large due to a scatter in the data from the various measurements.

The dipole moment was determined from Stark shift measurements by using a precision de voltage source (4) (Fluke, Model 413B). The effective guide spacing was determined using the $1 \rightarrow 2$ transition of OCS, $\mu=0.7152 \mathrm{D}(5)$.

\section{ANALYSIS AND DISCUSSION}

Ground state spectrum and structure. The transitions that were assigned for the ground vibrational state are listed in Table I. Only a type transitions were observed. The derived rotational constants and moments of inertia are listed in Table III. The rotational constants were obtained from a least-squares fit of the observed transitions. The uncertainties listed are twice the standard deviations.

Phosphabenzene is expected to be a planar molecule with the $a$ inertial axis being the $C_{2}$ symmetry axis. Because there are two pairs of equivalent hydrogen atoms, a 10:6 alternation of intensities is expected. The levels for which the $K$ value in the prolate limit is even will have the higher nuclear spin statistical weight. This alternation in intensity was clearly apparent for the closely spared $6_{06} \rightarrow 7_{07}$ and $6_{16} \rightarrow 7_{17}$ transitions and the $7_{07} \rightarrow 8_{08}$ and $7_{17} \rightarrow 8_{18}$ transitions.

Support for a planar conformation is obtained from the value of the inertial defect for the ground state. $I_{c}-I_{b}-I_{a}$ for phosphabenzene is $0.052 \mu \mathrm{A}^{2}$. This 
TABLE I

Measured Frequencies for Phosphabenzene (MHz)

\begin{tabular}{|c|c|c|c|c|c|c|c|c|}
\hline \multirow{2}{*}{ Transition } & \multicolumn{2}{|c|}{ Ground state } & \multicolumn{2}{|c|}{$V_{a}=1$ (antisym.) } & \multicolumn{2}{|c|}{$V_{b}=1(\mathrm{sym})$} & \multicolumn{2}{|c|}{$V_{c}=1$ (antisym) } \\
\hline & obsd & $\Delta \nu^{\mathrm{a}}$ & obsd & $\Delta \nu$ & obs. & $\Delta \nu$ & obsd & $\Delta \nu$ \\
\hline $3_{12} \rightarrow 4_{13}$ & 23380.36 & 0.05 & & & 23388.95 & 0.02 & & \\
\hline $3_{22} \rightarrow 4_{23}$ & 2183 & 0.05 & 21840.80 & 0.18 & & & & \\
\hline $4_{04} \rightarrow 5_{05}$ & 22912.49 & 0.07 & 22926.76 & 0.09 & 22930.87 & -0.09 & & \\
\hline $4_{14} \rightarrow 5_{15}$ & 22850.24 & -0.02 & 22864.68 & 0.14 & & & & \\
\hline $\mathbf{4}_{22} \rightarrow \mathbf{5}_{23}$ & 31257.95 & -0.05 & 31252.15 & -0.40 & 31271.05 & 0.05 & 31273.51 & -0.04 \\
\hline $4_{31} \rightarrow 5_{32}$ & 31207.09 & -0.00 & 31200.06 & 0.14 & 31228.97 & -0.03 & 31236.05 & 0.00 \\
\hline $5_{05} \rightarrow 6_{06}$ & 27045.58 & -0.03 & 27063.00 & -0.65 & 27069.14 & 0.58 & 27066.20 & -0.39 \\
\hline $5_{15} \rightarrow 6_{10}$ & 27028.84 & -0.03 & 2704 & 0.02 & 27051.55 & -0.41 & 27050.15 & 0.07 \\
\hline $5_{14} \rightarrow 6_{15}$ & 31478.47 & -0.02 & 31487.26 & 0.07 & 31493.06 & -0.04 & 31490.63 & 0.02 \\
\hline $5_{24} \rightarrow 6_{25}$ & 31034.70 & 0.01 & 31043.7 & 0.06 & 31053.16 & 0.00 & & \\
\hline $6_{06} \rightarrow 7_{07}$ & 31196.57 & -0.05 & 31218.54 & 0.12 & 31224.00 & -0.01 & 31221.55 & -0.18 \\
\hline $6_{16} \rightarrow 7_{17}$ & 31192.44 & 0.04 & 31214.36 & 0.16 & 31219.87 & 0.01 & 31217.60 & 0.00 \\
\hline $6_{15} \rightarrow 7_{16}$ & 35471.74 & -0.01 & 35484.57 & 0.16 & 35491.72 & -0.08 & 35489.29 & -0.08 \\
\hline $6_{25} \rightarrow 7_{26}$ & 35315.55 & 0.01 & 35328.30 & 0.09 & 35337.38 & 0.00 & 35335.90 & 0.17 \\
\hline $7_{07} \rightarrow 8_{08}$ & 35352.94 & -0.03 & 35378.47 & -0.07 & 35384.76 & 0.00 & 35382.30 & 0.16 \\
\hline $7_{17} \rightarrow 8_{18}$ & 35351.97 & 0.00 & 35377.43 & -0.10 & 35383.65 & -0.12 & 35381.31 & 0.15 \\
\hline
\end{tabular}

${ }^{a} \Delta \nu=\nu$ (observed) $-\nu$ (calculated)

is in the range expected for a planar conformation and can be compared with other compounds containing aromatic rings (6): pyridine, $0.032 \mu \mathrm{A}^{2}$; fluorobenzene, $0.027 \mu \mathrm{A}^{2}$; benzonitrile, $0.073 \mu \mathrm{A}^{2}$ and furan $0.048 \mu \mathrm{A}^{2}$. By analogy with these compounds and from the regularity of the vibrational satellite data described below, the expected planar configuration is indicated.

There are five structural parameters which define the ring geometry for phosphabenzene. It is possible to estimate two of these parameters using the $I_{a}$ and $I_{b}$ moments of inertia and assumptions for the other parameters and the positions of the hydrogens. These assumptions were guided by the structural parameters of other aromatic compounds and from those determined from $\mathrm{X}$-ray investigations of 1,5-dimethyl, 3-phenyl phosphabenzene (I), 1,1-dimethoxy-2,4,6triphenylphosphabenzene (II) and 2-t-butyl-4-aryl-5, 6 dihydronaptho-1,2-phosphorin (III) $(7-9)$. The CH bonds were assumed to be $1.08 \AA$ and to bisect their adjacent ring angles. The two $\mathrm{CC}$ bounds were set at $1.40 \AA$; while $<C_{2} C_{3} C_{4}=$ $122^{\circ}$ and $\angle C_{1} C_{2} C_{3}=123.6^{\circ}$ were assumed. This gave the following results: $d(\mathrm{PC})=1.727 \AA\left\langle C_{1} P C_{5}=101.1^{\circ}\right.$ and $\angle P C_{1} C_{2}=125^{\circ}$. This ring structure is nearly identical to that obtained for I except for the $2^{\circ}$ larger CPC angle in the substituted phosphabenzene. The PC bond distance is also close to that in II although the PCC angle was larger $\left(107^{\circ}\right)$ and $<C_{1} C_{2} C_{3}$ was smaller $\left(120^{\circ}\right)$ in that compound. In III, $d(\mathrm{PC})$ was $1.75 \AA$ and $<\mathrm{CPC}$ was $103^{\circ}$.

The meaning of the derived parameters must be qualified since they will vary 
TABLE II

Measured Frequencies for Phosphabenzene ( $\mathrm{MHz}$ )

\begin{tabular}{|c|c|c|c|c|c|c|c|c|}
\hline \multirow{2}{*}{ Transition } & \multicolumn{2}{|c|}{$V_{d}=1$ (antisym.) } & \multicolumn{2}{|c|}{$V_{a}=2(\mathrm{sym})}$. & \multicolumn{2}{|c|}{$V_{b}=2(\mathrm{sym})$} & \multicolumn{2}{|c|}{$\begin{array}{c}V_{a}=V_{b}=1 \\
(\text { antisym.) }\end{array}$} \\
\hline & obsd & $\Delta \nu$ & obsd & $\Delta \nu$ & obsd & $\Delta \nu$ & obsd & $\Delta \nu$ \\
\hline $\begin{array}{l}4_{22} \rightarrow 5_{2.3} \\
4_{31} \rightarrow 5_{32}\end{array}$ & 31260.49 & -0.09 & $\begin{array}{l}31247.41 \\
31192.60\end{array}$ & $\begin{array}{r}0.06 \\
-0.03\end{array}$ & 31283.51 & 0.08 & 31266.29 & 0.00 \\
\hline $5_{05} \rightarrow 6_{00}$ & 27055.24 & -0.24 & 27081.73 & 0.12 & 27090.97 & 0.17 & 27086.64 & 0.16 \\
\hline $5_{15} \rightarrow 6_{16}$ & 27038.75 & 0.17 & 27064.24 & -0.55 & 27074.25 & -0.15 & 27069.69 & -0.19 \\
\hline $5_{14} \rightarrow 6_{1 j}$ & 31488.10 & -0.06 & 31496.14 & -0.07 & 31507.21 & 0.13 & 31502.00 & 0.00 \\
\hline $5_{24} \rightarrow 6_{25}$ & 31043.11 & 0.29 & & & 31070.80 & -0.14 & & \\
\hline $\begin{array}{l}6_{06} \rightarrow 7_{07} \\
6_{15} \rightarrow 7_{16}\end{array}$ & 35482.70 & -0.06 & 31240.20 & 0.09 & & & & \\
\hline $6_{25} \rightarrow 7_{20}$ & 35325.80 & 0.02 & 35341.10 & 0.06 & 35358.25 & -0.18 & & \\
\hline $7_{07} \rightarrow 8_{08}$ & 35366.24 & 0.01 & 35404.04 & 0.09 & 35415.58 & -0.01 & 35410.09 & -0.02 \\
\hline $7_{17} \rightarrow 8_{18}$ & 35365.17 & -0.05 & 35403.08 & 0.14 & 35414.73 & 0.11 & 35409.16 & 0.04 \\
\hline
\end{tabular}

as the assumptions involving the carbon atoms are changed. In order to obtain longer PC bonds with our data, the $\mathrm{CC}$ bonds would have to be shortened below values reported for benzene derivatives. This suggests that a value of $1.73 \AA$ as an upper limit for the PC bond length in phosphabenzenc. A lower limit of $1.70 \AA$ can be set by assuming that the $\mathrm{CC}$ bonds will not be larger than $1.44 \AA$ (about 0.04-0.05 $\AA$ longer than in typical benzene derivatives, pyridine and the substituted phosphabenzenes). Hence, our data are most consistent with a PC bond of $1.70-1.73 \AA$ and $<\mathrm{CPC}$ of $101^{\circ}-104^{\circ}$. This $\mathrm{PC}$ bond length is betwe'n that found in $\left(\mathrm{CH}_{3}\right)_{3} \mathrm{P}(1.843 \AA)$ and the double bond in $\left(\mathrm{C}_{6} \mathrm{H}_{5}\right)_{3} \mathrm{P}=\mathrm{CH}_{2}(1.66 \AA)$ $(10,11)$.

Vibrational satellite spectra. The ground state transitions were accompanied by a number of weaker transitions originating from excited vibrational states. Seven states were identified based on their rigid rotor frequency fit and their Stark effects. The assigned rotational transitions are in Tables I and II. The rotational constants derived by a least-square fit and the inertial defects are listed in Table III.

From intensities and the changes in rotational constants, it was concluded that these vibrational satellites arose from four fundamental modes of vibration. The spectra were characterized by two intense satellites, $\nu_{a}$ and $\nu_{b}$, and two much weakcr states, $v_{c}$ and $v_{d}$. The other states observed were $2 \nu_{a}, 2 \nu_{b}$ and several transitions from $\nu_{a}+v_{b}$.

The transitions, $7_{07} \rightarrow 7_{08}$ and $7_{17} \rightarrow 7_{18}$, are separated by about $1 \mathrm{MHz}$. The intensities of these transitions were used to detcrmine whether the spin statistic were the same or reversed from the ground state. States having the same spin statistics as the ground state were $\nu_{b}, 2 v_{b}$ and $2 \nu_{a}$. The remaining states showed a reversal. IIence, it was concluded that $\nu_{a}, \nu_{c}$ and $\nu_{d}$ are fundamental modes antisymmetrie to the $C_{2}$ axis while $\nu_{b}$ is symmetric. 
TABLE III

Rotational Constants ( $M H z$ ), Moments of Inertra $\left(\mu \AA^{2}\right)$ and Inertial Defects $\left(\mu \AA^{2}\right)$ for Phosphabenzene

\begin{tabular}{lrrrrrrr}
\hline & \multicolumn{1}{c}{$A$} & \multicolumn{1}{c}{$B$} & \multicolumn{1}{c}{$C$} & \multicolumn{1}{c}{$I_{a}$} & \multicolumn{1}{c}{$I_{b}$} & $I_{0}$ & $\Delta^{a}$ \\
\hline Ground state & 5113.93 & 3505.54 & 2079.39 & 98.8234 & 144.1649 & 243.0405 & 0.0522 \\
& +0.04 & \pm 0.01 & \pm 0.01 & & & & \\
$V_{a}=1$ & 5109.74 & 3504.03 & 2081.27 & 98.9044 & 144.2270 & 242.8210 & -0.3104 \\
& \pm 0.26 & \pm 0.04 & \pm 0.02 & & & & \\
$V_{b}=1$ & 5108.82 & 3506.89 & 2081.57 & 98.9223 & 144.1096 & 242.7860 & -0.2459 \\
& \pm 0.22 & \pm 0.04 & \pm 0.02 & & & & \\
$V_{c}=1$ & 5107.49 & 3507.49 & 2081.40 & 98.9480 & 144.0849 & 242.8061 & -0.2268 \\
& \pm 0.20 & \pm 0.03 & \pm 0.02 & & & & \\
$V_{d}=1$ & 5115.47 & 3505.38 & 2080.25 & 98.7939 & 144.1714 & 242.9402 & -0.0251 \\
& \pm 0.28 & \pm 0.06 & \pm 0.02 & & & & \\
$V_{a}=2$ & 5105.87 & 3502.56 & 2083.13 & 98.9793 & 144.2878 & 242.6037 & -0.6634 \\
$V_{b}=2$ & \pm 0.30 & \pm 0.05 & \pm 0.02 & & & & \\
& 5103.72 & 3508.18 & 2083.69 & 99.0211 & 144.0563 & 242.5388 & -0.5386 \\
$V_{a}=V_{b}=1$ & \pm 0.30 & \pm 0.06 & \pm 0.02 & & & & \\
& 5104.80 & 3505.50 & 2083.13 & 99.0001 & 144.1666 & 242.5681 & -0.5995 \\
& \pm 0.30 & \pm 0.05 & \pm 0.02 & & & & \\
\hline
\end{tabular}

a $\Delta=I_{\mathrm{c}}-I_{b}-I_{a} ;$ conversion factor; $505376 \mathrm{MHz} \cdot \mu \AA^{2}$.

The vibrational frequencies of the two lowest modes were estimated by measuring their intensities relative to the ground state as described in the experimental section. This gave the values, $\nu_{a}=290 \pm 40 \mathrm{~cm}^{-1}$ and $\nu_{b}=325 \pm 40 \mathrm{~cm}^{-1}$. The remaining five states were estimated to lie in the range $450-650 \mathrm{~cm}^{-1}$. They were too weak, however, to obtain more meaningful vibrational frequencies except that $\nu_{c}$ was noted to have a lower frequency than $\nu_{d}$. Although no ir data in this region are yet available for comparison, we plan a complete study of the vibrational spectrum.

Some assignments of these modes are suggested by correlation with the low frequency modes in pyridine $(12,13)$. The two lowesi modes in pyridine, $\nu_{15 a}\left(A_{2}\right)$, $374 \mathrm{~cm}^{-1}$ and $\nu_{16 b}\left(B_{2}\right) 304 \mathrm{~cm}^{-1}$ are associated with $\nu_{b}$ and $\nu_{a}$, respectively, in phosphabenzene. Their nuclear spin statistics and negative inertial defects are consistent with this. The spin statistics for $\nu_{c}$ and $\nu_{d}$ indicate antisymmetric states. The analogous low-frequency modes in pyridine of this symetry are: $\nu_{6 a}\left(B_{1}\right)$, $605 \mathrm{~cm}^{-1} ; \nu_{1}\left(B_{2}\right), 675 \mathrm{~cm}^{-1}(11)$ or $749 \mathrm{~m}^{-1}(12)$; and $\nu_{11}\left(B_{2}\right), 700 \mathrm{~cm}^{-1}$. It is not possible to assign $\nu_{c}$ and $\nu_{d}$ unambiguously among these alternatives. The negative inertial defects do not necessarily eliminate $\nu_{6 a}$ from consideration since this state could have a negative inertial defect, especially if anothcr out-of-plane mode is close to it $(6)$.

Dipole moment. The dipole moment was determined from the Stark shift of the three transitions listed in Table IV. These data gave a dipole moment of $1.54 \pm 0.02 \mathrm{D}$. Interestingly this is identical to the dipole moment reported for 
TABLE IV

Stark Coefficients ( $\mathrm{MHzV}^{-2} \mathrm{~cm}^{2}$ ) and Dipole Moment for Phosphabenzene

\begin{tabular}{crr}
\hline Transition & $\Delta \nu / \epsilon^{2}$ obsd & \multicolumn{1}{c}{$\Delta \nu / \epsilon^{2}$ calcd } \\
\hline $3_{21} \rightarrow 4_{22} M=1$ & $-4.20 \times 10^{-6}$ & $-4.19 \times 10^{-6}$ \\
$3_{22} \rightarrow 4_{23} M=1$ & $+5.15 \times 10^{-6}$ & $5.12 \times 10^{-6}$ \\
$4_{22} \rightarrow 5_{23} M=2$ & $-1.94 \times 10^{-6}$ & $-1.95 \times 10^{-6}$ \\
& $|\mu|=1.54 \pm 0.02 \mathrm{D}$ & \\
\hline
\end{tabular}

2,4,6-triphenyl phosphabenzene (14). The direction of the dipole moment along the symmetry axis can not be determined from these data. Oehling and Schweig using the CNDO/2 formalism, calculated the charge distribution in phosphabenzene and pyridine (15). They concluded that the negative end of the dipole is oriented in the same direction in both compounds, viz., towards the hetero atom.

\section{CONCLUSION}

The microwave spectrum of phosphabenzenc? is consistent with the interpretalion that the phosphorus atom participates in $\pi$ conjugation with the carbon atoms. The molecule is planar with $C_{2 v}$ symmetry. The low frequency vibrational modes, when compared with pyridine, are reasonable for a conjugated planar species. Finally, while a unambiguous value could not be determined, the limits placed on the PC bond length are indicative of some double bond character.

\section{ACKNOWLEDGMENTS}

This work was supported by grants (GP-28155) and (GP-30589X) from the National Science Foundation. We are grateful to Professor Richard Schwendeman at Michigan State University for the use of his Hewlett-Packard spectrometer and for providing a computer program which aided in the structure calculations.

Rhchiven: November 22, 1971

\section{REFERENCES}

1. A. J. Ashe III, J. Amer. Chem. Soc. 93, 3293 (1971).

2. R. L. Kuczkowski, J. Amer. Chem. Soc. 90, 1705 (1968).

3. A. Esbitt and E. B. Wilson, Jr., Rev. Sci. Instr. 34, 901 (1963).

4. W. H. Kirchнoff, J. Amer. Chem. Soc. 90, 1705 (1968).

5. J. S. Muenter, J. Chem. Phys. 48, 4544 (1968).

6. D. R. Herschback and V. W. Laurie, J. Chem. Phys. 40, 3142 (1964).

7. J. C. J. Bart and J. J. Daly, Angew. Chem. Int. Ed. 7, 811 (1968).

8. U. Thewalt, Angew. Chem. Int. Ed. 8, 769 (1969).

9. W. Fischer, E. Hellner, S. Chatzidakis ind K. Dimroth, Tetrahedron Lett. 6227 (1968).

10. P. S. Bryan and R. L. Kuczkowski, J. Chem. Phys. 55, 3049 (1971).

11. J. C. J. BART, J. Chem. Soc. London B 350 (1969).

12. L. Corssin, B. J. Fox And R. C. Lord, I. Chem. Phys. 21, 1170 (1953).

13. J. K. Wilmhurst and H. J. Bernstein, Can. J. Chem. 35, 1183 (1957).

14. K. Dimroth And W. StÄde, Angew. Chem. 80, 966 (1968).

15. H. Oehling ANd A. Schweig, Tetrahedron Tett. 4941 (1970). 